\title{
Accurate checks of universality for Dyson's hierarchical model
}

\author{
J. J. Godina \\ Departamento de Física, CINVESTAV-IPN, Apartado Postal 14-740, Mexico, Distrito Federal 07000 \\ and Department of Physics and Astronomy, University of Iowa, Iowa City, Iowa 52242 \\ Y. Meurice and M. B. Oktay \\ CERN, 1211 Geneva 23, Switzerland \\ and Department of Physics and Astronomy, University of Iowa, Iowa City, Iowa 52242*
}

(Received 5 February 1998; published 8 May 1998)

\begin{abstract}
Using recently developed methods, we perform high-accuracy calculations of the susceptibility near $\beta_{c}$ for the $D=3$ version of Dyson's hierarchical model. Using linear fits, we estimate the leading $(\gamma)$ and subleading $(\Delta)$ exponents. Independent estimates are obtained by calculating the first two eigenvalues of the linearized renormalization group transformation. We found $\gamma=1.29914073 \pm 10^{-8}$ and $\Delta=0.4259469 \pm 10^{-7}$ independently of the choice of local integration measure (Ising or Landau-Ginzburg). After a suitable rescaling, the approximate fixed points for a large class of local measure coincide accurately with a fixed point constructed by Koch and Wittwer. [S0556-2821(98)50311-6]
\end{abstract}

PACS number(s): 05.50.+q, 11.10.Hi, 75.40.Cx

Scalar field theory has many important applications such as superconductivity, low energy descriptions of quarkantiquark bound states and possibly the mechanism responsible for the mass generation of all the experimentally observed particles. However, there exists no approximate treatment of this theory which could pretend to compete in accuracy with quantum electrodynamics at low energy, where renormalized perturbation theory can be used to calculate the magnetic moment of the electron and the muon with more than ten significant digits. Given the inherent difficulties associated with the experimental probing of very short distances, accurate calculations compared with accurate "low-energy", experiments may become more of a standard procedure in the next century.

In order to obtain a completely satisfactory treatment of scalar field theory in various dimensions, one needs an approximation scheme such that (a) the zeroth-order approximation preserves the main qualitative features of the model, (b) the zeroth-order approximation allows very accurate calculations, and (c) the zeroth-order approximation can be improved systematically and in a way which preserves its computational advantages. We advocate here that hierarchical approximations such as the approximate recursion formula derived by Wilson [1] or the related recursion formula which holds for Dyson's hierarchical model [2] could be used as such a zeroth-order approximation. The fact that the approximate recursion formula satisfies the requirement (a) is discussed at length in Ref. [1]. The fact that requirement (b) is satisfied in the symmetric phase is explained in Refs. [3, 4] and briefly reviewed below. The use of the hierarchical approximation solves some important problems encountered in practical calculations in lattice field theory. First, it possible to perform all the integrations appearing in the calculation of the zero-momentum Green's function in a much more efficient way than with the Monte Carlo procedure. Second, the

\footnotetext{
*Present address.
}

computing time scales only like the log of the number of sites, and one can eliminate finite-size effects completely. This can justify the effort of trying to solve part (c) of the above program which is a very difficult problem.

Wilson's approximate recursion formula is closely related to the recursion formula appearing in Dyson's hierarchical model [2]. Both models have no wave function renormalization $(\eta=0)$. It is possible to interpolate continuously between the two models and to show that during this process, the critical exponent associated with the susceptibility $\gamma$ varies [5] by less than 5 percent with respect to the nearest neighbor value. However, the numerical treatment of the two models is completely identical. In the following, we specialize the discussion to the case of Dyson's model because this model has been studied [3,6-10] in great detail in the past. We want to make clear that this choice is not essential and anything done below could have been done for Wilson's approximate formula.

In a typical lattice field calculation, we pick some values for the bare parameters entering in an action and we calculate the renormalized quantities. In general, the physical masses cannot be too large, when expressed in cutoff units. Ideally, we should be able to cover a broad range of situations going from effective theories with a low cutoff (e.g. $\left(m_{\rho} / m_{\pi}\right) \simeq 6$ for an effective theory of pions) to a "fundamental" theory with a large cutoff and which requires some fine-tuning procedure. In the following calculations, the fixed bare parameters will appear in a local measure of the Landau-Ginzburg (LG) form:

$$
W_{0}(\phi) \propto e^{-\left[(1 / 2) m^{2} \phi^{2}+g \phi^{2 p}\right]} .
$$

The limit [1] of a large UV cutoff $\Lambda$, in units of the physical mass $m_{R}$, can be reached by tuning another parameter $\beta$, which is the inverse temperature in Dyson's formulation of the model [2]. More explicitly, $\Lambda / m_{R}$ is proportional to $\left(\beta_{c}-\beta\right)^{-\gamma / 2}$. 
By fine-tuning $\beta$, one can approach a fixed point of the renormalization group (RG) transformation and describe the renormalization group ( $\mathrm{RG}$ ) flows using the linear approximation. As we will show later, for $\beta$ close enough to $\beta_{c}$ (i.e., for $\Lambda$ large enough), one can approximate very well the magnetic susceptibility (zero-momentum two point function) with a linearized expression taking into account only the first irrelevant direction:

$$
\chi \simeq\left(\beta_{c}-\beta\right)^{-\gamma}\left[A_{0}+A_{1}\left(\beta_{c}-\beta\right)^{\Delta}\right] .
$$

If we were sure that there is only one non-trivial fixed point (universality) and if we could calculate accurately the exponents, then the complicated procedure described above can be reduced to the determination of $A_{0}$ and $A_{1}$ in Eq. (2), a procedure that involves no fine-tuning.

In this Rapid Communication, we provide empirical evidence suggesting that the RG transformation of Dyson's hierarchical model has only one non-trivial fixed point. We calculate the exponents $\gamma$ and $\Delta$ with two independent methods (direct fit and linearization). The accuracy of our results is significantly better than the accuracy reached in the past $[3,6,7,9]$. All the approximate fixed points we have constructed below are very close (after rescalings explained below) to the fixed point calculated with an extraordinary accuracy by Koch and Wittwer [8]. Our work demonstrates the enormous calculational advantage of using the hierarchical approximation and addresses the question of understanding to what extent expansions about a known fixed point can be used as a substitute to the lengthy calculations in terms of bare parameters described above.

For the sake of completeness, we briefly review the steps which lead to the basic expression of the RG transformation of Eq. (6). The block-spin transformation of the hierarchical model is an integral formula which transforms the local measure $W(\phi)$ according to the rule:

$$
\begin{aligned}
& W_{n+1}(\phi) \propto e^{(\beta / 2)(c / 4)^{n+1} \phi^{2}} \\
& \quad \times \int d \phi^{\prime} W_{n}\left(\frac{\left(\phi-\phi^{\prime}\right)}{2}\right) W_{n}\left(\frac{\left(\phi+\phi^{\prime}\right)}{2}\right),
\end{aligned}
$$

where $c=2^{1-2 / D}$ in order to approximate $D$-dimensional nearest neighbor models. For more details, the reader may consult Refs. [4, 8, 9]. In the following we only consider the case $D=3$ in the symmetric phase. We approach criticality for a fixed initial $W_{0}$ by fine-tuning $\beta$ as described in Ref. [4]. When a critical value $\beta_{c}$ is reached approximately, a (discrete) scale invariance is temporarily restored and it is convenient to reabsorb the scale factor $(c / 4)$ in $\phi^{2}$. After this rescaling, we obtain the conventional $\mathrm{RG}$ transformation of the local measure. In Fourier form it reads

$$
R_{n+1}(k)=C_{n+1} \exp \left(-\frac{1}{2} \beta \frac{\partial^{2}}{\partial k^{2}}\right)\left[R_{n}\left(\frac{k \sqrt{c}}{2}\right)\right]^{2}
$$

We fix the normalization constant $C_{n}$ in such way that $R_{n}(0)=1$. We consider the finite dimensional approximations of degree $l_{\text {max }}$ :

$$
R_{n}(k)=1+a_{n, 1} k^{2}+a_{n, 2} k^{4}+\cdots+a_{n, l_{\max }} k^{2 l_{\max }} .
$$

The coefficients $a_{n, l}$ are proportional $[4,10,9]$ to the expectation value of the sum of all the fields (after $n$ iterations, there are $2^{n}$ of them) denoted $M_{n}$. In particular, the finite volume susceptibility $\chi_{n}(\beta)$, defined as $\left\langle\left(M_{n}\right)^{2}\right\rangle_{n} / 2^{n}$ is simply $-2 a_{n, 1}(2 / c)^{n}$. When $\beta<\beta_{c}, \chi_{n}$ reaches a finite limit $\chi$, when $n$ goes to infinity. The recursion formula for $a_{n, m}$ is purely algebraic:

$$
a_{n+1, m}=\frac{\sum_{l=m}^{l_{\max }}\left(\sum_{p+q=l} a_{n, p} a_{n, q}\right)[(2 l) ! /(l-m) !(2 m) !](c / 4)^{l}[-(1 / 2) \beta]^{l-m}}{\sum_{l=0}^{l_{\max }}\left(\sum_{p+q=l} a_{n, p} a_{n, q}\right)[(2 l) ! / l !](c / 4)^{l}[-(1 / 2) \beta]^{l}} .
$$

The initial condition for the Ising measure is $R_{0}=\cos (k)$. For the Landau-Ginsburg measure, the coefficients in the $k$-expansion need to be evaluated numerically.

In a recent article [4], we have shown that the errors on $\chi$ due to finite volume and finite truncations fell exponentially fast with, respectively, the number of iterations used and the dimension of the truncated space $\left(l_{\max }\right)$. It is possible to make calculations, where these errors play no practical role. The main limitation of the method comes from the round-off errors which are amplified, when many iterations are spent near the fixed point. If the arithmetic operations are performed with a precision $\delta$, then [4]

$$
\left|\frac{\delta \chi}{\chi}\right| \sim \frac{\delta}{\beta_{c}-\beta} \text {. }
$$

We now proceed to determine the values of the four pa- rameters appearing in Eq. (2) from direct calculations of $\chi$ at various temperatures. The calculations which follow have been performed for two particular choices of $W_{0}$, one corresponding to the Ising limit $\left[W_{0}(\phi)=\delta\left(\phi^{2}-1\right)\right]$ and the other to the choice $m^{2}=1, p=2$, and $g=0.1$ in Eq. (1). Unless specified differently, the calculations are performed using double-precision. In the following, we use the notation $x$ for the quantity $-\log _{10}\left(\beta_{c}-\beta\right)$. If we display $\log (\chi)$ versus $x$, the deviations from the linear behavior are not visible to the eye and need to be studied and understood "locally" in $\beta$. In order to get a rough understanding of the corrections, we have divided the computer data in 14 bins of 100 points. The first bin contains data for values of $x=1.00,1.01, \ldots$ 1.99 and so on. In each bin (indexed $i$ ), we make a linear fit of $\log _{10}(\chi)$ versus $x$. In the $i$ th bin, we call the slope $\gamma^{(i)}$, and $\left(\sigma^{(i)}\right)^{2}$ denotes the sum of the squares of the difference 


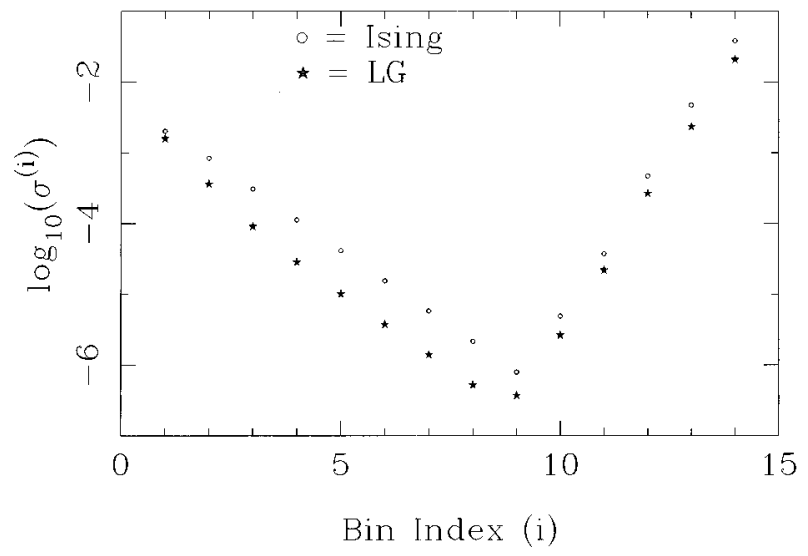

FIG. 1. The deviations from the linear fits $\sigma^{(i)}$ defined in the text as functions of the bins, for the Ising model (circles) and the Landau-Ginzburg model (stars).

between the data and the linear fit divided by the number of points in a bin (100) minus 2. The values of $\sigma^{(i)}$ are displayed in Fig. 1. This graph can be interpreted easily. There are two known sources of deviations from the linear behavior: the subleading corrections to the scaling laws (which decrease when $\beta$ gets close to $\beta_{c}$ ) and the round-off errors [which increase, when $\beta$ gets close to $\beta_{c}$ according to Eq. (7)]. The approximate slopes in Fig. 1 confirm this interpretation. In bin 9, we minimize the combined deviations from linearity and we can consider $\gamma^{(9)}$ as a first estimate of $\gamma$. Its numerical value is 1.29917 in the Ising case and 1.29914 in the Landau-Ginzburg (LG) case. With this simple procedure, we have already gained almost two significant digits compared to the existing estimates [3,7,9], where the answer $\gamma$ $=1.300$ was consistently obtained with errors of order 1 in the last digit.

We can improve this result by estimating the subleading corrections. For this purpose, we have used the bins 6 and 7, where the next subleading corrections are small (see discussion later) and where the numerical errors are still not too large. We have divided these two bins into 10 sub-bins of 100 points each. We use two digit indices for these sub-bins. For instance sub-bin 6.3 is the third sub-bin of bin 6 and contains the values of $x: 6.3,6.301, \ldots, 6.399$. Using Eq. (2), the same kind of notations as above for $\gamma$ and noting that $j+0.0495$ is the middle of the sub-bin indexed by $j$, we obtain the approximate decay law:

$$
\gamma^{(j)} \simeq \gamma-\Delta\left(\frac{A_{1}}{A_{0}}\right) 10^{-\Delta(j+0.0495)} .
$$

The unknown coefficients can be extracted from linear fits of $\log _{10}\left(\gamma^{(j+0.1)}-\gamma^{(j)}\right)$. We obtained $A_{1} / A_{0}=-0.57$ and $\Delta$ $=0.428$ for the Ising model and $A_{1} / A_{0}=0.14$ and $\Delta$ $=0.427$ for the LG model specified above. Repeating the first step (a linear fit in bin 9), but with $\chi$ divided by [1 $\left.+\left(A_{1} / A_{0}\right)\left(\beta_{c}-\beta\right)^{\Delta}\right]$, we obtain $\gamma=1.299141$ with an agreement up to the sixth decimal place between the two models considered above.

Equation (7) is an unavoidable limitation if we use double precision arithmetic. However, using MATHEMATICA with a suitably set precision, $l_{\max }=42$ for the Ising model and $l_{\max }$
$=50$ for the LG model (see Ref. [4] for the determination of these quantities), we were able to calculate $\chi$ in bins 10,11 , and 12 with 11 significant digits. In the following, we call this data the "high-precision data." Since this procedure is relatively lengthy, we have used only ten points per bins. We also determined $\beta_{c}$ with 24 significant digits so that in bin 12 , the subtracted quantity $\beta_{c}-\beta$ is also known with at least 11 significant digits. In the Ising case, the result is easily reproducible and reads $\beta_{c}=1.17903017044626973251189$. We have then used bin 12 (where the subleading corrections are very small and our errors on them are less important) with $\chi$ divided by the subleading correction as described above, to estimate $\gamma$. We then used this better value of $\gamma$ to obtain the subleading corrections in bin 7 (where they are more sizable). This procedure can be iterated. This "bootstrap" of linear fits converges rapidly. We reach a nine significant digit agreement between the high-precision data and the fit obtained with the above procedure. The small discrepancies can be analyzed in terms of first order errors made in the estimate of the four parameters. This linear analysis provides small corrections $\left(<4 \times 10^{-9}\right)$ to $\gamma$ and more sizable corrections $\left(<4 \times 10^{-4}\right)$ to $\Delta$. The size of these corrections provide an order magnitude estimate for the errors. After these small corrections are taken into account, we obtain an agreement between the exponents of the two models for the following digits: $\gamma=1.299140730$ and $\Delta=0.4260$. We conclude that $\gamma=1.29914073$ with an estimated error of less than $10^{-8}$.

We would like to comment about the corrections to Eq. (2) and how they could affect our estimates. First, since the third eigenvalue of the linearized RG transformation $\lambda_{3}$ $\simeq 0.48$, the next subleading exponent is approximately 2 . For $x>10$, these effects are negligible. Second, a general argument [1], suggests that we should replace the constant $A_{0}$ and $A_{1}$ in Eq. (2) by log-periodic function which can be expressed as linear superposition of Fourier modes of the form $\left(\beta_{c}-\beta\right)^{\left[i 2 \pi l / \ln \left(\lambda_{1}\right)\right]}$, with $l$ an integer. Evidence for nonzero Fourier modes were found in Ref. [9] by using an estimator of $\gamma-1$ called the extrapolated slope and denoted $\hat{S}_{m}$. In this estimator, oscillating and constant contributions have roughly the same amplitude. However, using Eqs. (3.7) to (3.10) of Ref. [9], one realizes that in $\hat{S}_{m}$, the oscillating amplitude is dramatically amplified by a factor of the order $\left|\omega^{3} / \Gamma(\gamma+i \omega)\right|$, where $\omega=2 \pi / \ln \left(\lambda_{1}\right) \simeq 18$. This implies that the Fourier coefficients of the non-zero modes are suppressed by at least 14 orders of magnitude. A direct search for these oscillations confirms this upper bound. Third, Eq. (2) is obtained from a linearization. Higher order corrections give contributions proportional to $\left(\beta_{c}-\beta\right)^{2 \Delta}$. An analysis of the difference between fit and data in low bins indicates that these corrections are the main source of errors in our analysis.

An alternative calculation of the exponents consists in linearizing the RG transformation near a fixed point. An approximate fixed point can be found by approaching $\beta_{c}$ from below with our best resolution and iterating until $a_{n+1,1} / a_{n, 1}$ takes a value which is as close as possible to 1 . In the present formulation, the linearized RG transformation is given by the $l_{\max } \times l_{\max }$ matrix 


$$
M_{l, m}=\frac{\partial a_{n+1, l}}{\partial a_{n, m}}
$$

evaluated at the (approximated) fixed point. Using the highprecision MATHEMATICA-based method described above, we obtained this approximate fixed point for $n=101$ for the Ising model and for $n=97$ for the LG model. Calculating the eigenvalues of Eq. (9) for the two models used for the first estimates, we obtain discrepancies of $2 \times 10^{-8}$ for $\lambda_{1}$ and of $4 \times 10^{-8}$ for $\lambda_{2}$. The average values are $\lambda_{1}=1.42717246$ and $\lambda_{2}=0.85941163$. Changing $n$ by one or improving the fixed point using Newton's method produce variations in these eigenvalues which are smaller than $3 \times 10^{-8}$. Using the relations $\gamma=2 \ln (2) / 3 \ln \left(\lambda_{1}\right)$ and $\Delta=-\ln \left(\lambda_{2}\right) / \ln \left(\lambda_{1}\right)$, we obtain $\gamma=1.29914078$ and $\Delta=0.4259469$ both with estimated errors of order $10^{-7}$. The new estimate of $\gamma$ is compatible with the previous one, but is less accurate. On the other hand, the new estimate of $\Delta$ is more accurate. The discrepancy with the previous estimate is less than $10^{-4}$ which is compatible with our previous error estimate.

The two approximated fixed points obtained in the above calculation depend on $\beta_{c}$. We denote them $R^{*}\left(k, \beta_{c}\right)$. However, it is possible to obtain what will turn out to be a universal function $U(k)$ by absorbing $\beta$ into $k$. More explicitly, we found that

$$
U(k)=R^{*}\left(\sqrt{\beta_{c}} k, \beta_{c}\right)
$$

is in very good approximation independent of the model considered. This function is related to a fixed point $f\left(s^{2}\right)$ constructed in Ref. [8] by the relation

$$
U(k) \propto f\left[\left(\frac{c-4}{2 c}\right) k^{2}\right] .
$$

The Taylor coefficients of $f$ can be found in the file approx.t in [8]. Normalizing Eq. (11) with $U(0)=1$, we obtain

$$
U(k)=1.0-0.358711349882 k^{2}+0.053537288227 k^{4}-\cdots .
$$

It is not known if there is only one non-trivial fixed point for Dyson's model. Both the two approximate fixed points we have constructed above give a function $U(k)$ very close to Eq. (12). The closeness can be characterized by the $\rho$-norms introduced in [8]. For $\rho=2$ and $l \leqslant 42$, we found that the error $\delta u_{l}$ on the $l$ th coefficients of the approximate $U(k)$ with respect to the accurate expression obtained from Ref. [8] were bounded by $\left|\delta u_{l}\right|<3 \times 10^{-8} / l ! 2^{l}$.

In order to further explore the possibility of having different fixed points, we have considered more LG models. Using the parametrization of Eq. (1), we have considered the 12 cases obtained by choosing among the following possibilities: $m^{2}= \pm 1$ (single or double-well potentials), $p=2,3$, or 4 (coupling constants of positive, zero and negative dimensions, when the cutoff is restored) and $g=10$ or 0.1 (moderately large and small couplings). These searches have been performed using regular double-precision calculations. We have not aimed at great accuracy. For all these twelve models, we found that using the same notations and conventions as a above $\left|\delta u_{l}\right|<5 \times 10^{-5} / l ! 2^{l}$. In other words, the function $U(k)$ seems to be independent of the general shape of the potential, the strength of the interactions and whether or not the model is perturbatively renormalizable.

In conclusion, our best estimates of the critical exponents $\gamma=1.29914073 \pm 10^{-8}$ and $\Delta=0.4259469 \pm 10^{-7}$ have an accuracy significantly better than existing estimates $[3,7,9]$. Our results demonstrate the power of the methods developed in Ref. [4]. They provide an incentive to develop more efficient perturbative calculations of the critical exponents and to attack the problem of the improvement of the hierarchical approximation. We found no indications for the existence of a non-trivial fixed point different from the one obtainable from Ref. [8]. Near criticality, or in field theoretical language for a large UV cutoff, the parametrization of Eq. (2) fits the data very well. The quantities $A_{0}$ and $A_{1}$ depend on the bare parameters in a complicated way. However, the fact that we can use confidently the universal features suggests that it is possible to shortcut the use of bare parameters and consider directly $A_{0}, A_{1}$ as an input. More generally, we are in position to check if the following conjecture is true: an expansion about the non-trivial fixed point can be used as a substitute for the calculations in terms of bare parameters. If true, this would mean that the result of Ref. [8] effectively "solves", the hierarchical model even far away from criticality.

This research was supported in part by the Department of Energy under Contract No. FG02-91ER40664. J. J. Godina is supported by CONACYT. Y. M. thanks P. Wittwer and the CERN lattice group for useful conversations.
[1] K. Wilson, Phys. Rev. B 4, 3185 (1971); Phys. Rev. D 3, 1818 (1971); K. Wilson and J. Kogut, Phys. Rep., Phys. Lett. 12C, 75 (1974); K. Wilson, Phys. Rev. D 6, 419 (1972).

[2] F. Dyson, Commun. Math. Phys. 12, 91 (1969); G. Baker, Phys. Rev. B 5, 2622 (1972).

[3] G. Baker and G. Golner, Phys. Rev. B 16, 2081 (1977).

[4] J. J. Godina, Y. Meurice, B. Oktay, and S. Niermann, Phys. Rev. D 57, 6326 (1998).

[5] Y. Meurice and G. Ordaz, J. Phys. A 29, L635 (1996).

[6] P. Bleher and Y. Sinai, Commun. Math. Phys. 45, 247 (1975);

P. Collet and J. P. Eckmann, ibid. 55, 67 (1977); H. Koch and
P. Wittwer, ibid. 106, 495 (1986); 138, 537 (1991); 164, 627 (1994).

[7] P. Collet, J.-P. Eckmann, and B. Hirsbrunner, Phys. Lett. 71B, 385 (1977).

[8] H. Koch and P. Wittwer, Math. Phys. Electr. J. 1, Paper 6 (1995) (http://mpej.unige.ch/).

[9] Y. Meurice, G. Ordaz, and V. G. J. Rodgers, Phys. Rev. Lett. 75, 4555 (1995); Y. Meurice, S. Niermann, and G. Ordaz, J. Stat. Phys. 87, 363 (1997).

[10] Y. Meurice and G. Ordaz, J. Stat. Phys. 82, 343 (1996). 